\title{
Simulação numérica do Processo Ecap (Prensagem em Canais Equiangulares) utilizando o aço VMB 300
}

\section{Numerical simulation of the Ecap Process (Equal Channel Angular Pressing) using the VMB 300 Steel}

Artigo

Original

\author{
Márcia Regina Baldissera ${ }^{1}$ \\ Paulo Rangel Rios ${ }^{2}$ \\ Jaime Pereira Gouvêa ${ }^{2}$ \\ Jefferson Fabrício Cardoso Lins ${ }^{2}$
}

Original

Paper

\begin{abstract}
Palavras-chaves: Resumo
ECAP $\quad$ O processo ECAP é uma técnica de deformação plástica severa, onde um tarugo é forçado a escoar numa matriz com dois canais de seções transversais

Elementos finitos

Deformação plástica severa. idênticas formando um determinado ângulo entre si. Uma das vantagens deste processo é que o material deformado não sofre alteração dimensional, além de poder ser processado em temperatura ambiente. A deformação do tarugo ocorre numa estreita zona localizada na intersecção entre os dois canais da matriz, sob condições de cisalhamento. Foram analisadas as distribuições das tensões e deformações causadas no material durante o processo, por meio de simulação numérica utilizando o programa ANSYS (versão 8.1) adotando condições isotérmicas e estado plano de deformação. Os resultados mostraram que as deformações ocorreram por cisalhamento num ângulo de $45^{\circ}$ próximo à intersecção dos canais. A parte inicial do tarugo não apresentou deformação, o que explica a necessidade de descartar esta região do material no término do processo.
\end{abstract}

\begin{abstract}
Equal channel angular pressing (ECAP) is one techniques of severe plastic deformation (SPD), where a billet forced to flow through a die containing two intersecting channels of identical cross-section. One of the advantages of this process is that the deformed materials maintain the original dimensions, in addition can be processed at room temperature. The deformation of the billet occurs in a small zone located at the intersection between the two channels of the die, under shear strain conditions. The stress and deformations distributions caused in the material during the process had been analyzed by means of numerical simulation, using the ANSYS program (version 8.1), adopting isothermal conditions and plane strain conditions. The results had shown that the deformations had occurred for shear in an angle of $45^{\circ}$ next to the intersection of the channels. The initial part of the billet did not present deformations, what it explains the necessity to discard this region of the material in the ending of the process.
\end{abstract}

Key words:

ECAP

Finite element simulation

Severe plastic deformation

\section{Introdução}

O processo de deformação plástica severa (DPS) provoca mudanças nas propriedades e na microestrutura dos materiais. Estas técni- cas são utilizadas para refinar grãos de metais até atingir granulometria ultrafina e/ou nanométrica. Este refino microestrutural inclui melhora nas propriedades mecânicas, óticas, elétricas e magnéticas ${ }^{1,2}$. A vantagem deste

${ }_{1}^{1}$ Doutoranda - Universidade Federal Fluminense - Escola de Engenharia Industrial Metalúrgica de Volta Redonda
${ }^{2}$ Docente - Universidade Federal Fluminense - Escola de Engenharia Industrial Metalúrgica de Volta Redonda 
processo é produção de material em "bulk" facilitando o estudo do comportamento mecânico em relação à nova microestrutura ultrafina. Em processos de DPS convencionais como estiramento e/ou laminação as dimensões são reduzidas com o aumento da deformação, gerando materiais na forma de fios ou filamentos, limitando suas aplicações industriais ${ }^{3}$.

O processo ECAP, inventado por Segal ${ }^{4}$, é um método simples de prensagem que provoca deformação plástica por cisalhamento em um tarugo que se move através de uma matriz contendo dois canais de igual seção transversal ${ }^{1,5}$, acumulando elevados níveis de deformação plástica $(\varepsilon>7)$. A figura 1 ilustra este processo. Os canais são conectados e usualmente perfazem um ângulo de $90^{\circ}$ entre si, sendo que este ângulo pode variar de acordo com a dificuldade de deformação dos materiais ${ }^{3,6}$. Em condições ideais o tarugo é deformado numa estreita área de deformação localizada na intersecção dos canais.

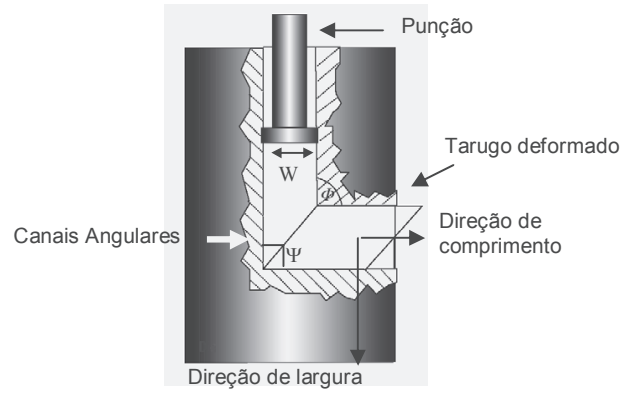

Figura 1: Desenho esquemático de um processo $\mathrm{ECAP}^{7}$.

O método de elementos finitos (FEM) é amplamente utilizado para analisar o comportamento da deformação em um material processo por ECAP. Este método utiliza alguns dados de entrada como: condições de contorno não linear, propriedades do material e condições de carregamento para analisar parâmetros como, o coeficiente de atrito e a variação dos ângulos dos canais da matriz e encontrar condições ótimas de processo para um dado material ${ }^{(1)}$.

\section{Materiais e Métodos}

A simulação por FEM foi realizada no estado plano de deformação bidimensional em temperatura ambiente, usando o programa ANSYS (versão 8.1). A matriz foi considerada como um corpo rígido com os seguintes parâ- metros geométricos: $\Phi=90^{\circ}$ e $\Psi=0^{\circ}$ e o tarugo de seção retangular de 15,0 mm de comprimento e 5,0 mm de largura. A curva tensão versus deformação verdadeira do aço VMB $300^{(8)}$ foi usada para descrever as características do material. O coeficiente de atrito utilizado no contato entre o tarugo e a matriz foi de 0,1 .

\section{Resultados e Discussão}

Durante o processo de DPS foram identificados os pontos críticos de tensão e deformação causados no aço VMB 300. A maior concentração de tensão se dá ao longo de um ângulo de $45^{\circ}$ formado entre os canais da matriz, como é mostrado na figura 2. Neste modelo a tensão máxima equivalente (von Mises) encontrada foi de $840 \mathrm{MPa}$, o que de acordo com as propriedades mecânicas do material encontra-se na região plástica. $\mathrm{O}$ comportamento das tensões cisalhantes (XY) mostra que o tarugo sofre deformação por cisalhamento ao passar pela intersecção dos canais da matriz, enquanto na análise das tensões na direção X mostra maior concentração na base inferior do tarugo, indicando o arraste de material em direção ao segundo canal.

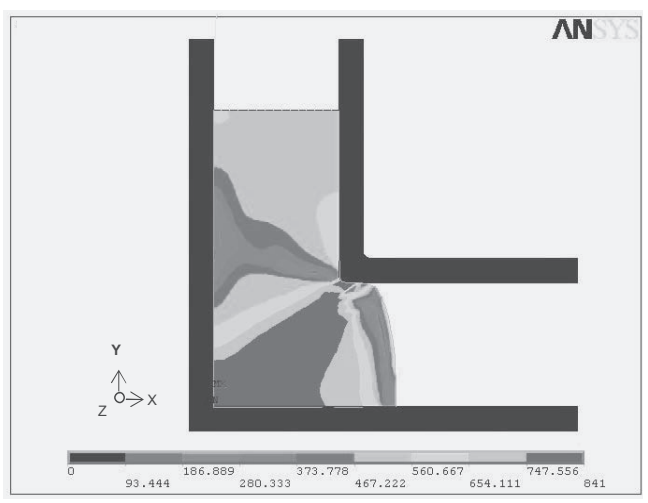

Figura 2: Distribuição das tensões de von Mises no tarugo.

A figura 3 mostra que há uma distribuição de deformação plástica cisalhante no tarugo perfazendo um ângulo de $45^{\circ}$ entre os canais. Foi observada também uma concentração significativa de deformação na base inferior do tarugo, causando uma grande distorção da malha nesta região dificultando o avanço do processo de prensagem. A distorção da malha pode ser relacionada com a deformação dos grãos do material, comprovando a possibilidade de modelar o processo de refino microestrutural através de elementos finitos. 


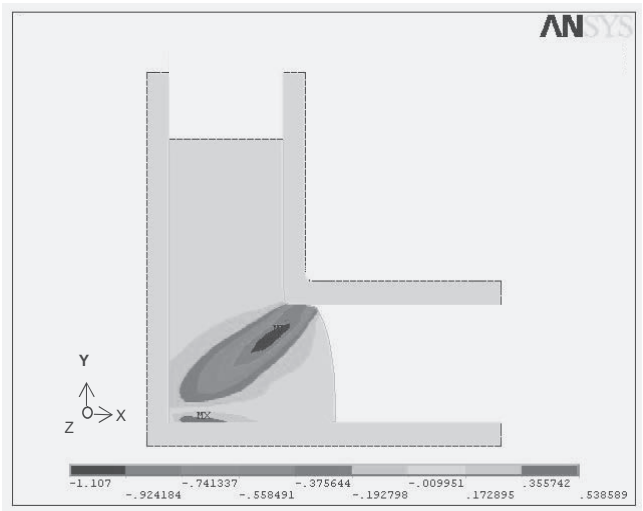

Figura 3: Distribuição das deformações plásticas por cisalhamento no tarugo.

A figura 4 mostra as deformações na direção $X$, onde na região circulada é zero. Nesta figura observou um ponto máximo no canto inferior do tarugo indicando a possibilidade de ocorrer um descolamento entre o tarugo e a matriz. Foi observado que a superfície frontal do tarugo não sofreu deformação plástica, não mostrando distorção na malha, pois no início do processo esta região não foi submetida às ações das tensões cisalhantes, não sofrendo deformação. Esta região deve ser descartada ao analisar as deformações.

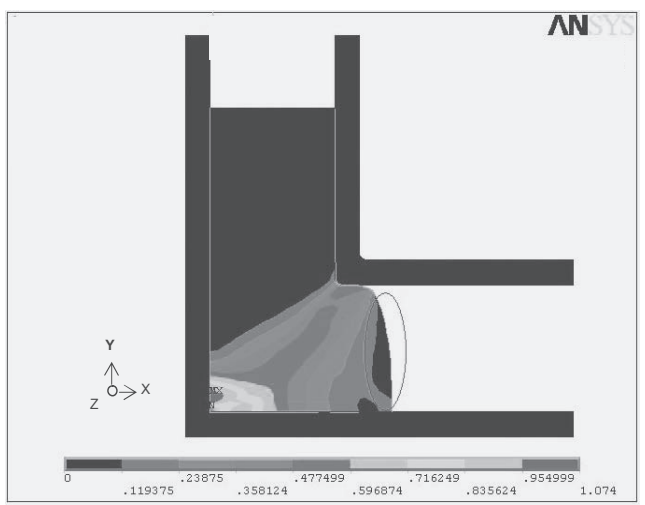

Figura 4: Distribuição da deformação plástica na direção $\mathrm{X}$ durante o processo ECAP.

Através da simulação numérica foi realizada a análise da evolução das tensões e deformações plásticas na região mais solicitada do tarugo durante o processo. O gráfico da figura 5 mostra as tensões na região de intersecção dos canais da matriz. As tensões equivalente (von Mises) e nas direções $\mathrm{Y}$ e $\mathrm{Z}$ aumentam em direção à base inferior do tarugo onde atingem seu ápice. As tensões na direções $\mathrm{X}$ e cisalhante (XY) não apresentam grandes oscilações no percurso da análise, isto porque a região observada abrange todos os valores máximos das respectivas tensões alcançadas no processo. A distribuição das deformações plásticas logo após a intersecção dos canais é mostrada na figura 6 , onde as deformações nas direções X, Y, cisalhante (XY) e equivalente aumentam continuamente, porém com intensidades diferentes, até atingirem o máximo localizado na base inferior do tarugo.

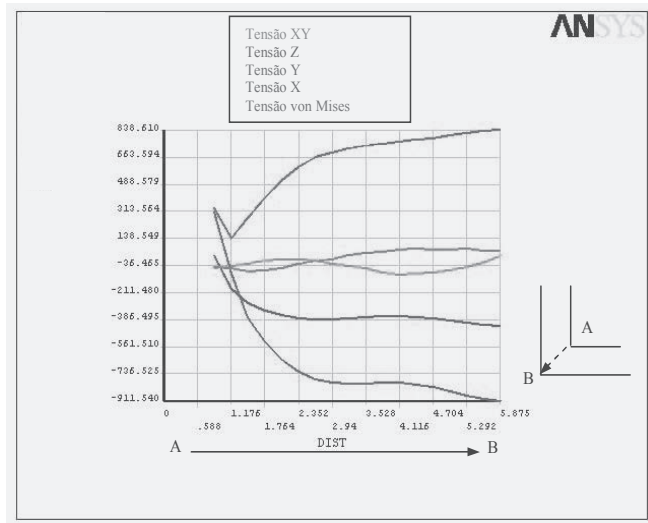

Figura 5: Distribuição das tensões do tarugo na região de intersecção da matriz.

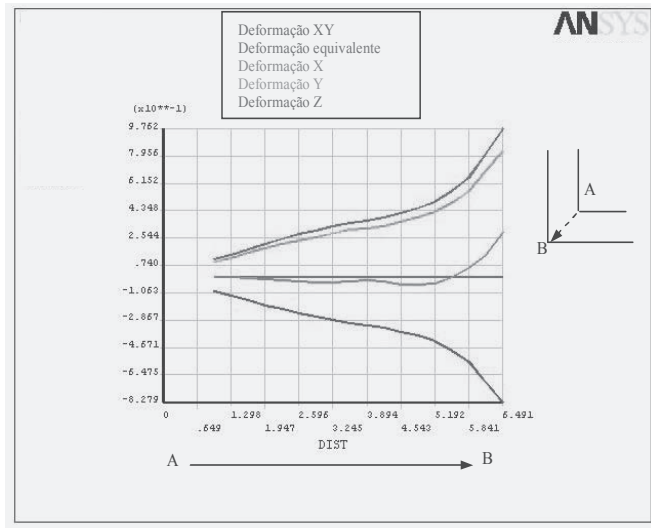

Figura 6: Distribuição das deformações plásticas do tarugo na região de intersecção da matriz.

Considerando que o material é isotrópico e o processo apresenta continuidade, o que acontece em um ponto do tarugo após passar pela zona cisalhante é o mesmo que irá acontecer num ponto subseqüente, assim foi possível realizar uma distribuição das tensões e deformações ao longo do movimento do tarugo pelos canais da matriz. Os gráficos de tensão cisalhante (máxima e mínima) e tensão equivalente (von Mises) é apresentado na figura 7 (a) e (b). A faixa de tensão cisalhante aumenta até atingir um pico quando o tarugo é movimentado cerca de 2,2 $\mathrm{mm}$ em direção ao segundo canal da matriz, em seguida encontra-se um estado quase estacionário que não ultrapassam 480 $\mathrm{MPa}$. Isto indica que o material já sofreu a 
ação máxima da tensão cisalhante imposta pelo processo ECAP. Este comportamento pode ser visualizado no gráfico da tensão de von Mises onde ocorre um rápido aumento até movimentar o tarugo cerca de $2,0 \mathrm{~mm}$ e em seguida atinge um estado estacionário com tensão máxima de $841 \mathrm{MPa}$. A estabilidade das tensões mostra que a partir deste ponto máximo o material já passou pelo máximo de deformação plástica imposto pelo processo.


Figura 7: Comportamento (a) das tensões cisalhantes (máx. e mín.) e (b) tensão de von Mises em função do movimento do tarugo.

A figura 8 mostra a distribuição das deformações equivalentes ao longo do movimento do tarugo. Foi encontrado que a faixa de deformação equivalente (máxima e mínima) aumenta à medida que o material passa para $\mathrm{o}$ segundo canal da matriz. A faixa de deformação equivalente $(\varepsilon)$ está compreendida entre 0,7 e 1,10, o que concorda com a deformação equivalente máxima $(\varepsilon=1,15)$ reportado na literatura ${ }^{(1)}$ para um passe de prensagem numa matriz com a mesma configuração geométrica. Este resultados de deformação equivalente, juntamente com os resultados da tensão de von Mises indicam que o tarugo já passou pela zona de máxima deformação imposta pelo processo ECAP.

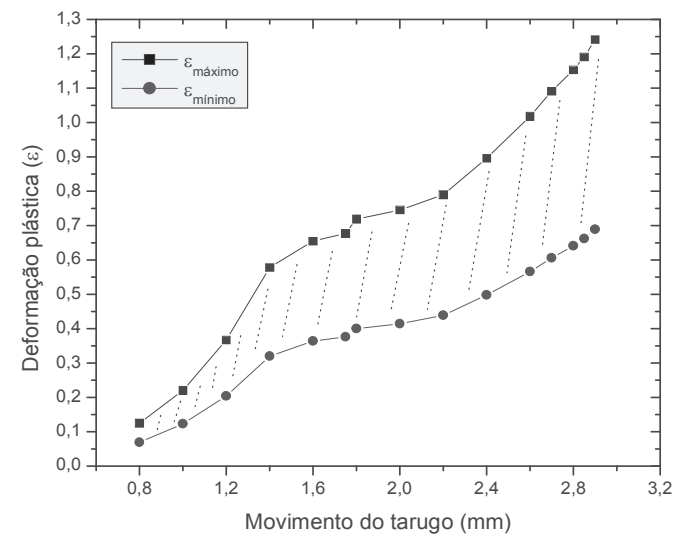

Figura 8: Comportamento da deformação equivalente (máx. e mín.) em função do movimento do tarugo.

\section{Conclusões}

Os resultados mostraram que as tensões e as deformações estão localizadas ao longo da intersecção dos canais perfazendo um ângulo de $45^{\circ}$. Através da distribuição da deformação plástica na direção $\mathrm{X}$, verificou-se a possibilidade de abertura entre o tarugo e a matriz, devido à concentração de tensões no canto inferior do material. Não foi possível realizar a passagem total do tarugo para o segundo canal devido à grande distorção ocasionada na malha, a qual pode ser facilitada pela readequação da malha a cada movimento do tarugo e pela limitação do valor da força de atrito. Entretanto, o movimento imposto ao tarugo neste trabalho já foi suficiente para confirmar a região crítica de distribuição de tensões e deformações, que o processo ECAP impõe ao material.

\section{Agradecimentos}

Este trabalho teve o suporte financeiro da Coordenação de Aperfeiçoamento de Pessoa de Nível Superior, CAPES.

\section{Referências Bibliográficas}

1. KIM, H.S., SEO, M.H., HONG, S.I., Finite element analysis of equal channel angular pressing of strain rate sensitive metals, Journal of Materials Processing Technology, vol. 130131, pp. 497-503, 2002. 
2. HORITA, Z., FUJINAMI, T., et al., Improvement of mechanical properties for $\mathrm{Al}$

alloys using equal-channel angular pressing, Journal of Materials Processing Technology, vol. 117, pp. 288-292, 2001.

3. BOWEN, J.R., GHOLINIA, A., ROBERTS, S.M., PRANGNEL, P.B., Analysis of the billet deformation behavior in equal channel angular extrusion. Materials Science and Engineering A, vol. A287, pp. 87-99, 2000.

4. SEGAL, V.M., Engineering and commercialization of equal channel angular extrusion (ECAE). Materials Science and Engineering A, vol. 386, pp. 269-276, 2004.

5. KIM, H.S., ESTRIN,Y., Microstructural modelling of equal channel angular pressing for producing ultrafine grained materials. Materials Science and Engineering A, 2005.

6. PRANGNELL, P.B., HARRIS, C. and ROBERTS, S.M., Finite element modeling of equal channel angular extrusion. Scripta Materialia, vol. 37, nº. 7, pp. 983-989, 1997.

7. VALIEV, R.Z., Nanostructuring of metals by severe plastic deformation for advanced properties. Nature Materials, v.3, pp.511-516, 2004

8. SOUZA, V.B.F., Modelamento e simulação do processo de conformação de tubos de seção quadrangular, 2004, Dissertação de Mestrado (Mestrado em Metalurgia) - Escola de Engenharia Industrial Metalúrgica de Volta Redonda-UFF, Volta Redonda-RJ.

Endereço para Correspondência:

Márcia Regina Baldissera

Universidade Federal Fluminense, Escola de Engenharia Industrial Metalúrgica de Volta Redonda marciab@metal.eeimvr.uff.br

Avenida dos trabalhadores, 420

Vila Santa Cecília - Volta Redonda - RJ

CEP: 27255-125

Informações bibliográficas:

Conforme a NBR 6023:2002 da Associação Brasileira de Normas Técnicas (ABNT), este texto científico publicado em periódico eletrônico deve ser citado da seguinte forma: BALDISSERA, Márcia Regina; RIOS, Paulo Rangel; GOUVÊA, Jaime Pereira; LINS, Jefferson Fabrício Cardoso; Simulação numérica do Processo Ecap (Prensagem em Canai Equiangulares) utilizando o aço VMB 300. Cadernos UniFOA. Volta Redonda, ano IV, n. 11, dezembro 2009. Disponível em: < http://www.unifoa.edu.br/cadernos/edicao/11/29.pdf $>$ 